\title{
TAUBERIAN THEOREMS FOR SUMMABILITY TRANSFORMS
}

\author{
ROHITHA GOONATILAKE
}

Received 11 February 2004 and in revised form 9 July 2004

The convolution summability method is introduced as a generalization of the randomwalk method. In this paper, two well-known summability analogs concerning the strong law of large numbers (SLLN) and the law of the single logarithm (LSL), that gives the rate of convergence in SLLN for the random-walk method, are extended to this generalized method.

\section{Introduction}

Let $A=\left[a_{n, k}\right]$ for $n, k \geq 0$ be an infinite matrix of (complex) numbers. We say that a sequence $\left\{s_{i}\right\}_{i \geq 0}$ is $A$-summable to $s$ (summable to $s$ by the $A$ method) if the series

$$
(A s)_{n}:=\sum_{k=0}^{\infty} s_{k} a_{n, k} \longrightarrow s \quad(n \longrightarrow \infty)
$$

we then write $s_{n} \rightarrow s(A)$, where $A$ is the $A$ method of summability. Appropriate choices of $A=\left[a_{n, k}\right]$ for $n, k \geq 0$ give the classical methods [2]. In this paper, we present various summability analogs of the strong law of large numbers (SLLN) and their rates of convergence in an unified setting, beyond the class of random-walk methods. A convolution summability method introduced in the next section as an extension of the random-walk method generalizes the classical Euler, Borel, Taylor, and Meyer-König type matrix methods [16]. This corresponds to the distribution of sums of independent and identically distributed (i.i.d.) integer-valued random variables. The identically distributed condition is relaxed to some extent in the convolution summability method to include other summability methods.

Chow [5] gave the following analog of almost sure convergence of the Borel $B$ and the Euler $E_{p}$ methods of summability to $\mu$ requiring the finiteness of the second moment for sequence of i.i.d. random variables. 
Theorem 1.1 (Chow [5]). If $Z,\left\{Z_{i}\right\}_{i \geq 1}$ is a sequence of i.i.d. random variables, then the following statements are equivalent:

(1) $\operatorname{Var}(Z)<\infty, E Z=\mu$,

(2) $Z_{n} \rightarrow \mu(B)$ a.s., where $B$ is the Borel method,

(3) $Z_{n} \rightarrow \mu\left(E_{p}\right)$ for some (all) $p \in(0,1)$, a.s., where $E_{p}$ is the Euler method with parameter $p$.

A similar characterization has been considered for the Cesàro method of order $\alpha>1$ and the Abel method of summability [17]. Issues involving the values of $\alpha$ in the Cesàro method are found in $[3,6,8,10,12,13,14,17,18]$.

A summability matrix transformation, $R_{n, k}=P\left(S_{n}=k\right)$ for $n, k \geq 0$, where $S_{n}=X_{1}+$ $X_{2}+\cdots+X_{n}$ is the sum of nonnegative integer-valued random variables, is called the random-walk method. Later Bingham and Maejima [4] extended the above theorem to include the random-walk method as follows.

Theorem 1.2 (Bingham and Maejima [4]). If $Z,\left\{Z_{i}\right\}_{i \geq 1}$ forms a sequence of i.i.d. random variables, then the following statement is equivalent to (1), (2), and (3) and each of those items are equivalent to each other as stated in Theorem 1.1:

(4) $Z_{n} \rightarrow \mu(R)$, where $R$ is the random-walk method generated by a sequence of independent nonnegative aperiodic integer-valued random variables with positive variance and finite third moment.

Theorem 1.2 is now extended to a larger class, the convolution summability method.

\section{Preliminaries}

Much of our discussion will now revolve around the following types of summability methods. This is a larger class of summability methods that includes random-walk method and many others.

Definition 2.1. Let $\left\{p_{k}\right\}_{k \geq 0}$ and $\left\{q_{k}\right\}_{k \geq 0}$ be two sequences of nonnegative numbers with $\sum_{k=0}^{\infty} p_{k}=1$ and $\sum_{k=0}^{\infty} q_{k}=1$. Define a summability matrix, $C=\left[C_{n, k}\right]$, whose entries are given by $C_{0, k}=q_{k}$ and $C_{n+1, k}:=\left(C_{n} \text {. * } p\right)_{k}=\sum_{j=0}^{k} p_{j} C_{n, k-j}$ for $n, k \geq 0$. The matrix $C$ is called a convolution summability matrix.

A useful probabilistic interpretation of $C$ is the following. Let $Y, X_{1}, X_{2}, \ldots$ be a sequence of independent nonnegative integer-valued random variables such that $Y$ has probability function $q$ and the $X_{i}$ 's are identically distributed with probability function $p$. Let $S_{0}=Y$ and $S_{n}=Y+X_{1}+\cdots+X_{n}$ for $n \geq 1$. Let $\left\{p_{j}\right\}_{j \geq 0}$ and $\left\{q_{j}\right\}_{j \geq 0}$ be the probability distributions of $X_{1}, X_{2}, \ldots$ and $Y$, respectively. The $n$th row and $k$ th column entry of the convolution summability matrix $C$ is the probability $C_{n, k}=P\left(S_{n}=k\right)$. The method $C$ is regular if and only if $P\left(X_{1}=0\right)<1$ [16]. Some classical summability methods are examples of the method $C$. For instance, when $Y=0$ and $X_{1} \sim \operatorname{Binomial}(1,1 / 2)$ (Bernoulli with $p=1 / 2$ ), then $C$ becomes the Euler method denoted by $E_{r}$. When $Y \sim$ $X_{1} \sim$ Poisson(1), we get the Borel matrix method. When $Y \sim \operatorname{Geometric}(1-r)$ and $X_{1} \sim$ $Y+1$, then we get the Taylor method. And when $Y \sim X_{1} \sim \operatorname{Geometric}(1-r)$, we get 
the Meyer-König method. We shall call $C$ a convolution method and when $Y=0$ with probability 1 , it is called the random-walk method. The method $C$ can be extended to nonidentically distributed random variables; however, it will serve our purpose adequately as it is. Hence, it is suspected that the regular convolution summability matrix transformation $\left[C_{n, k}\right]$ for $n, k \geq 0$ obeys this theorem. The regular convolution summability matrix referred to everywhere in this paper has the above construction with appropriate moment conditions.

It is known that the Borel method and the random-walk method are comparable for bounded sequences [2]. Stemming from this fact, in comparing the random-walk method with the convolution summability method, we expect them to obey the same type of Tauberian condition, which eventually paves the way to the equivalence of these two methods. Under similar conditions, we will prove appropriate extensions to the theorems of Bingham and Maejima [4] with the assumptions of finite positive variance and third moment. This convolution summability method has a particular choice of weights, namely, if the random-walk method is generated by a sequence of i.i.d. aperiodic nonnegative integer-valued random variables $\left\{X_{i}\right\}_{i \geq 1}$ with finite third moment, then the convolution method is considered to be generated by $Y,\left\{X_{i}\right\}_{i \geq 1}$, where $\left\{X_{i}\right\}_{i \geq 1}$ is the same as before and $Y$ a nonnegative integer-valued random variable independent of $\left\{X_{i}\right\}_{i \geq 1}$. Further, they correspond to probability functions $\{p\}$ and $\{q\}$ of the convolution summability method, respectively. We certainly impose some moment conditions on $Y$ as the case may be. Any comparison between the random-walk method and the convolution summability method that will be executed in this paper will be in the above setting. Of course, the methods are assumed to be strongly regular, so that $\operatorname{Var}\left(X_{1}\right)>0$.

The random-walk method can be considered as a particular convolution summability method. The corresponding $\{p\}$ and $\{q\}$ sequences are then of the form: $q=(1,0,0, \ldots)$ and $p=\left(p_{0}, p_{1}, p_{2}, \ldots\right)$.

\section{Tauberian theorems}

Theorems of the type in which ordinary convergence is deduced from the fact that one has some type of summability condition and perhaps, an additional condition are called Tauberian theorems. On the other hand, the conditions on the sequences for which two summability methods are equivalent are studied by another type of Tauberian theorem.

We now compare the random-walk method and the convolution summability method subject to a given Tauberian condition with the appropriate assumptions on the moments of random variables. Several other results immediately follow from this result. We state them as corollaries to the main result.

We first need a lemma.

Lemma 3.1. For every fixed integer $j>0$, it follows that

$$
\sum_{k=0}^{\infty} \sqrt{k}\left|P\left(S_{n}=k\right)-P\left(S_{n}=k+j\right)\right|=j O(1) .
$$

Proof. The proof is accomplished by the principle of mathematical induction. 
If $j=1$, then we have

$$
\sum_{k=0}^{\infty} \sqrt{k}\left|P\left(S_{n}=k\right)-P\left(S_{n}=k+1\right)\right| \leq \kappa
$$

for some $\kappa<\infty$ which follows from [1, Theorem 2].

Suppose that the result holds true for some $j>1$, that is,

$$
\sum_{k=0}^{\infty} \sqrt{k}\left|P\left(S_{n}=k\right)-P\left(S_{n}=k+j\right)\right| \leq j \kappa .
$$

The result for $j \rightarrow j+1$ is

$$
\begin{aligned}
\sum_{k=0}^{\infty} & \sqrt{k}\left|P\left(S_{n}=k\right)-P\left(S_{n}=k+j+1\right)\right| \\
\leq & \sum_{k=0}^{\infty} \sqrt{k}\left|P\left(S_{n}=k\right)-P\left(S_{n}=k+j\right)\right| \\
& +\sum_{k=0}^{\infty} \sqrt{k}\left|P\left(S_{n}=k+j\right)-P\left(S_{n}=k+j+1\right)\right| \\
\leq & j \kappa+\kappa=(j+1) \kappa .
\end{aligned}
$$

The next two theorems, Theorems 3.2 and 3.3, show that the random-walk method and the convolution summability method are equivalent after assuming integrability condition on the random variable $Y$.

Theorem 3.2. Let the random-walk method be generated by a sequence of independent identically distributed aperiodic nonnegative integer-valued random variables $\left\{X_{i}\right\}_{i \geq 0}$ with finite third moment and positive variance and the convolution method is considered to be generated by $Y,\left\{X_{i}\right\}_{i \geq 0}$, with $\left\{X_{i}\right\}_{i \geq 0}$ the same as before and $Y$ a nonnegative integervalued random variable independent of $\left\{X_{i}\right\}_{i \geq 0}$ with $E|Y|^{3 / 2}<\infty$. Then for a given sequence $\left\{f_{i}\right\}_{i \geq 0}$, which obeys the Tauberian condition $f_{k}=o(\sqrt{k})$ for all $k \geq 1$, the random-walk method and the convolution summability method are equivalent.

Theorem 3.2 has a longer proof [11] that heavily depends on repeated applications of the triangle inequality; telescopic effects of a series; [11, Lemmas 1,2, and 3]; interchange of summations; essentially moving absolute values in and out of the sums; and Bikjalis and Jasjunas [1, Theorem 2]. However, Theorem 3.2 with somewhat weakened assumptions, namely, $0<\operatorname{Var}\left(X_{1}\right)<\infty$ and $E|Y|<\infty$ has the shortest proof and follows in a few lines from Lemma 3.1. An estimate for Theorem 3.2 and the proof of Theorem 3.3 have been provided to the author through personal communication with H. Kesten in 2001. The theorem is therefore stated with the weakest assumptions and the shortest proof for those who are just interested to know the results at their best. Theorem 3.2 now easily follows from this revised version of the theorem. 
Theorem 3.3. Let the random-walk method be generated by a sequence of independent identically distributed aperiodic nonnegative integer-valued random variables $\left\{X_{i}\right\}_{i \geq 0}$ with finite third moment and positive variance and the convolution method is considered to be generated by $Y,\left\{X_{i}\right\}_{i \geq 0}$, with $\left\{X_{i}\right\}_{i \geq 0}$ the same as before and $Y$ a nonnegative integer-valued random variable independent of $\left\{X_{i}\right\}_{i \geq 0}$ with $E|Y|<\infty$. Then for a given sequence $\left\{f_{i}\right\}_{i \geq 0}$, which obeys the Tauberian condition $f_{k}=o(\sqrt{k})$ for all $k \geq 1$, the random-walk method and the convolution summability method are equivalent.

Proof. Note first that Lemma 3.1 implies that (in a somewhat relaxed notation)

$$
\begin{aligned}
& \sum_{k=0}^{\infty} o(\sqrt{k})\left|P\left\{S_{n}=k\right\}-P\left\{S_{n}=k-j\right\}\right| \\
& \leq \sum_{\ell=0} \sum_{k=0}^{\infty} o(\sqrt{k})\left|P\left\{S_{n}=k-\ell\right\}-P\left\{S_{n}=k-\ell-1\right\}\right| \\
& \leq \sum_{\ell=0}^{j-1} \sum_{k=0}^{\infty} o(\sqrt{(k+\ell)})\left|P\left\{S_{n}=k\right\}-P\left\{S_{n}=k-1\right\}\right| \\
& \leq \sum_{\ell=0} \sum_{k=0}^{\infty} o(\sqrt{k}+\sqrt{\ell})\left|P\left\{S_{n}=k\right\}-P\left\{S_{n}=k-1\right\}\right| \\
& \leq \sum_{\ell=0}\left[o(1)+o\left(\frac{\sqrt{\ell}}{\sqrt{n}}\right)\right] \\
&= j o(1)+o\left(\frac{j^{3 / 2}}{\sqrt{n}}\right)
\end{aligned}
$$

as $n \rightarrow \infty$ as an easy estimate for Theorem 3.2. In the last step but one of (3.5) the following, (3.6) is used

$$
\sum_{k=-\infty}^{\infty}\left|P\left\{S_{n}=k\right\}-P\left\{S_{n}=k-1\right\}\right|=O\left(\frac{1}{\sqrt{n}}\right) .
$$

This follows from [1, Theorem 2] or [15, Theorem 3]. In addition, one also has, as $n \rightarrow \infty$,

$$
\begin{aligned}
& \sum_{k=0}^{\infty} o(\sqrt{k})\left|P\left\{S_{n}=k\right\}-P\left\{S_{n}=k-j\right\}\right| \\
& \quad \leq \sum_{k=0}^{\infty}\left[o(\sqrt{k}) P\left\{S_{n}=k\right\}+o(\sqrt{k}) P\left\{S_{n}=k-j\right\}\right] \\
& \quad=o(1)+o\left(E \sqrt{S_{n}}\right)+\sum_{k=0}^{\infty} o(\sqrt{k+j}) P\left\{S_{n}=k\right\} \\
& \quad \leq o(\sqrt{n})+\sum_{k=0}^{\infty} o(\sqrt{k}+\sqrt{j}) P\left\{S_{n}=k\right\} \\
& \quad \leq o(\sqrt{n})+o(\sqrt{j}) .
\end{aligned}
$$


Consequently

$$
\begin{aligned}
\left|\sum_{k=0}^{\infty} C_{n, k} f_{k}-\sum_{k=0}^{\infty} R_{n, k} f_{k}\right| & \leq \sum_{k=0}^{\infty}\left|f_{k}\right| \sum_{j=0}^{\infty} q_{j}\left|P\left\{S_{n}=k-j\right\}-P\left\{S_{n}=k\right\}\right| \\
& \leq \sum_{j=0}^{n} q_{j} j o(1)+\sum_{j>n} q_{j} o(\sqrt{j})=o(1) .
\end{aligned}
$$

This means that the sequence $\left\{f_{i}\right\}_{i \geq 1}$ is summable to $L$ by the random-walk method. This completes the proof of the required assertion.

Remark 3.4. In conclusion, all members of the convolution summability methods; Euler, Borel, Taylor, Meyer-König methods, and so forth are equivalent to their corresponding random-walk method for a sequence $\{f(k)\}_{k \geq 0}$ with $f_{k}=o(\sqrt{k})$.

Since

$$
E\left[|Y|^{3 / 2}\right]<\infty, \quad f_{k}=O(1) \Longrightarrow E[|Y|]<\infty, \quad f_{k}=O(\sqrt{k})
$$

the following two corollaries are trivial consequences of Theorem 3.3.

COROLlaRY 3.5. Let the random-walk method be generated by a sequence of independent identically distributed nonnegative aperiodic integer-valued random variables $\left\{X_{i}\right\}_{i \geq 0}$ with finite third moment and $\operatorname{Var}\left(X_{1}\right)>0$ and the convolution method is considered to be generated by $Y,\left\{X_{i}\right\}_{i \geq 0}$, with $\left\{X_{i}\right\}_{i \geq 0}$ is same as before, and $Y$ a nonnegative integer-valued random variable independent of $\left\{X_{i}\right\}_{i \geq 1}$ with $E|Y|<\infty$. Then for a bounded sequence, the random-walk method and the convolution summability method are equivalent.

Corollary 3.6. Let $\left\{X_{i}\right\}_{i \geq 1}$ be independent identically distributed random variables with finite third moment, $\operatorname{Var}\left(X_{1}\right)>0$, and let $\{Y, \hat{Y}\}$ be two random variables independent of $\left\{X_{i}\right\}_{i \geq 1}$ with $E|Y|, E|\hat{Y}|<\infty$. Then for a given sequence $\left\{f_{i}\right\}_{i \geq 0}$, which obeys the Tauberian condition $f_{k}=o(\sqrt{k})$ for all $k \geq 1$, two convolution methods generated by two sequences of independent aperiodic nonnegative integer-valued random variables $Y,\left\{X_{i}\right\}_{i \geq 1}$ and $\hat{Y}$, $\left\{X_{i}\right\}_{i \geq 1}$ are equivalent.

Borrowing the statement of [1, Theorem 2] and [15, Theorem 3], we could restate the relevant theorems in order to make the results more self-apparent. The appropriate statement for Theorem 3.3 is the following.

Theorem 3.7. Let the random-walk method be generated by a sequence of independent identically distributed integer-valued random variables $\left\{X_{i}\right\}_{i \geq 0}$ having distribution function $F$ and the convolution method is considered to be generated by $Y,\left\{X_{i}\right\}_{i \geq 0}$, with $\left\{X_{i}\right\}_{i \geq 0}$ the same as before, and $Y$ an integer-valued random variable independent of $\left\{X_{i}\right\}_{i \geq 1}$ with $E|Y|<\infty$. If, either

(a) $F$ is concentrated on the sequence $\{k+n h: n=0, \pm 1, \ldots\}$ for some $h>0$, then for each fixed integer $i$ with $P\left\{S_{i}-l k=i h\right\}>0$ for some $l$, or

(b) $F^{k}$ has an absolutely continuous component for some $k$, 
then for a given sequence, $\left\{f_{i}\right\}_{i \geq 0}$, which obeys the Tauberian condition $f_{k}=o(\sqrt{k})$ for all $k \geq 1$, the random-walk method and the convolution summability method are equivalent.

Remark 3.8. In conclusion, with above $F$, all members of the convolution summability methods; Euler, Borel, Taylor, Meyer-König methods, and so forth are equivalent to their corresponding random-walk method for a sequence $\{f(k)\}_{k \geq 0}$ with $f(k)=o(\sqrt{k})$.

\section{Almost sure convergence}

The results in the preceding sections will lead to the extension of the corresponding almost sure convergence analogue of Bingham and Maejima [4] to the convolution summability method of our choice with the assumption of the finite third moment of the random variable.

In pursuit of this, we prove the following theorem.

Theorem 4.1. Let $Z,\left\{Z_{i}\right\}_{i \geq 1}$ be a sequence of i.i.d. random variables with finite variance. Let $Y,\left\{X_{i}\right\}_{i \geq 1}$ with $E|Y|<\infty$ be independent and let $\left\{X_{i}\right\}_{i \geq 1}$ be i.i.d. aperiodic nonnegative integer-valued random variables with finite third absolute moment and $\operatorname{Var}\left(X_{1}\right)>0$. Denote $P(Y=k)=q_{k}$ and $P\left(X_{1}=k\right)=p_{k}$ and the resulting random-walk method by $R(p)$ and the convolution method by $C(p, q)$. Then the following are equivalent:

(I) $E Z=\mu, \operatorname{Var}(Z)<\infty$,

(II) $Z_{n} \rightarrow \mu$ a.s. $R(p)$ for $R$ some (any) random-walk method,

(III) $Z_{n} \rightarrow \mu$ a.s. $C(p, q)$ for $C$ some (any) convolution summability method.

Proof. (I) implies (II) and (II) implies (I) are already known [4]. Next, by the BorelCantelli lemma, we have $\operatorname{Var}(Z)<\infty$ if and only if $Z_{n}=o(\sqrt{n})$ a.s. So if (I) holds, then (II) holds by [4]. If $Z_{n}=o(\sqrt{n})$ a.s. and (II) holds, then by Theorem 3.2, (III) holds a.s. for a particular convolution summability method $C(p, q)$, where $R$ is generated by $\{p\}$. This gives that (I) implies (III) for a given method.

Finally, to show that (III) implies (I), we use the similar argument from [4]. Since

$$
P\left(\xi_{1}+\xi_{2}+\cdots+\xi_{n}=j\right)=\frac{1}{\tau(2 \pi n)^{1 / 2}} \exp \left\{-\frac{1}{2} \frac{(j-n \nu)^{2}}{n \tau^{2}}\right\}+o\left(\frac{1}{\sqrt{n}}\right)
$$

uniformly in $j$, where $\xi_{1}, \xi_{2}, \ldots, \xi_{n}, \ldots$ is a sequence of i.i.d. random variables with finite third moment [17], it follows for the convolution summability weights

$$
\begin{aligned}
C_{n, j} & =\sum_{k=0}^{j} q_{k} P\left(S_{n}=j-k\right) \\
& =\sum_{k=0}^{j} q_{k}\left\{\frac{1}{\tau(2 \pi n)^{1 / 2}} \exp \left\{-\frac{1}{2} \frac{(j-k-n \nu)^{2}}{n \tau^{2}}\right\}+o\left(\frac{1}{\sqrt{n}}\right)\right\} .
\end{aligned}
$$

This yields

$$
C_{n,[n v]} \sim \frac{1}{\tau(2 \pi n)^{1 / 2}}
$$


Our assumption is

$$
\sum_{j=0}^{\infty} C_{n, j} Z_{j} \longrightarrow \mu \quad \text { a.s., as } n \longrightarrow \infty .
$$

Write $Z_{j}^{s}$ for the symmetrization of $Z_{j}$. Then

$$
\sum_{j=0}^{\infty} C_{n, j} Z_{j}^{s} \longrightarrow 0 \quad \text { a.s., as } n \longrightarrow \infty .
$$

The above sum exists a.s. by the three-series theorem of Kolmogorov (see [7]). Put $Y_{n}=$ $\sum_{j \leq n \nu} C_{n, j} Z_{j}^{s}$ and $W_{n}=\sum_{j>n \nu} C_{n, j} Z_{j}^{s}$. Then $\left(Y_{1}, Y_{2}, \ldots, Y_{n}\right)$ and $W_{n}$ are independent for each $n$, and

$$
Y_{n}+W_{n} \longrightarrow 0 \quad \text { a.s., }
$$

so in probability. Since $Y_{n}$ and $W_{n}$ are independent and symmetric, we use the Lévy symmetrization inequalities [9, Chapter V.5, Lemma 2] to deduce

$$
Y_{n} \longrightarrow 0 \text { in probability. }
$$

Use of [5, Lemma 2] now yields

$$
Y_{n} \longrightarrow 0 \quad \text { a.s. }
$$

Recall that $Y_{n}$ is a sum over $j \leq \nu n$. Separate the last term of this sum from the previous terms, and apply the same argument to them as just applied to $W_{n}$ and $Y_{n}$. We have

$$
C_{n,[\nu n]} Z_{[v n]}^{s} \longrightarrow 0 \quad \text { a.s., as } n \longrightarrow \infty .
$$

Since $C_{n,[\nu n]} \sim 1 / \tau(2 \pi n)^{1 / 2}$, we obtain

$$
\frac{Z_{n}^{s}}{\sqrt{n}} \longrightarrow 0 \quad \text { a.s., as } n \longrightarrow \infty \text {. }
$$

By the Borel-Cantelli lemma, we obtain $E\left(\left|Z^{s}\right|^{2}\right)<\infty$. From

$$
E\left(\left|Z^{s}\right|^{2}\right)=\operatorname{Var}\left(Z^{s}\right)=2 \operatorname{Var}(Z)
$$

we deduce that $\operatorname{Var}(Z)<\infty$. Now (I) holds with $\mu=E Z$.

We know that (I) implies (II) for any random-walk method $R(p)$ with positive variance and finite third moment, where $\{p\}$ is the common distribution of $\left\{X_{i}, i \geq 1\right\}$. Then (II) implies (III), where $C(p, q)$ is the convolution method with $\sum_{j} j q_{j}<\infty(E|Y|<\infty)$. Then (III) implies (I). We show that any convolution method $C(s, q)$, where $\{s\}$ is not same as $\{p\}$, can also be used. By (I) and (II) we note that $R(p)$ can be replaced by $R(s)$. This then implies that $C(p, q)$ can be replaced by $C(s, q)$. This now completes the proof.

Remark 4.2. In conclusion, all members of the convolution summability methods; Euler, Borel, Taylor, Meyer-König methods and so forth obey the theorem of almost sure convergence. 


\section{Law of the single logarithm}

The classical law of the iterated logarithm gives us the rate at which the convergence in $Z_{n} \rightarrow \mu(C, 1)$ a.s. for a sequence of i.i.d. random variables takes place. Gaposhkin [10] has established the law of the iterated logarithm for the $(C, \alpha)$ and the Abel methods in the case, $Z_{1}$ is bounded. Actually Gaposhkin's results are extended by Lai [17] to the case, $0<E\left(Z_{1}\right)^{2}<\infty$. The extension is the sharpest possible in the sense that its converse also holds [17]. The primary interest in this section is to prove an analogue of the law of iterated logarithm already established for the random-walk method [4] for the convolution summability method. More precisely, we prove the law of the single logarithm (LSL) that contains the result of [4], as stated below.

The results derived from the preceding two sections will be utilized to extend this LSL [4] to the convolution summability method of given structure. It gives the rate at which the convergence in the previous theorems take place.

Theorem 5.1. Let $Z,\left\{Z_{i}\right\}_{i \geq 1}$ be a sequence of i.i.d. random variables with finite variance. For $Y,\left\{X_{i}\right\}_{i \geq 1}$ being independent with $E|Y|<\infty$ and $\left\{X_{i}\right\}_{i \geq 1}$ being i.i.d. aperiodic nonnegative integer-valued random variables with finite positive variance, $\sigma^{2}$ and third absolute moment, the following are equivalent:

(I) $E Z=0, \operatorname{Var}(Z)=d^{2} \in(0, \infty)$, and $E\left[Z^{4} /\left(1+\log ^{+}|Z|\right)^{2}\right]<\infty$;

(II) $\limsup _{n \rightarrow \infty}(4 \pi n)^{1 / 4}\left(d^{2} \log n\right)^{-1 / 2}\left|\sum_{j=0}^{\infty} R_{n, j} Z_{j}\right|=1 / \sigma^{1 / 2}$ a.s. for some (any) randomwalk method $R(p)$ or circle method $C$ with $E\left|X_{i}\right|^{3}<\infty$ for $i=1,2, \ldots$, where $R_{n, j}$ denotes the weights of the random-walk method $R(p)$ or circle method $C$;

(III) $\lim \sup _{n \rightarrow \infty}(4 \pi n)^{1 / 4}\left(d^{2} \log n\right)^{-1 / 2}\left|\sum_{j=0}^{\infty} C_{n, j} Z_{j}\right|=1 / \sigma^{1 / 2}$ a.s. for some (any) convolution summability method $C(p, q)$ with $E\left|X_{i}\right|^{3}<\infty$ for $i=1,2, \ldots$ and $E(Y)<\infty$, where $C_{n, j}$ denotes the weights of the convolution summability method $C(p, q)$.

Proof. (I) is equivalent to (II) by Bingham and Maejima [4]. The following argument is usually omitted, but given for the sake of completeness. As shown, the moment condition $E\left[Z^{4} /\left(1+\log ^{+}|Z|\right)^{2}\right]<\infty$ in (I) is equivalent to $Z_{j}=o\left(j^{1 / 4} \log ^{1 / 2} j\right)$ a.s. for all $j \geq 1$.

For brevity, denote the events $\left\{Z_{n}^{4} /\left(1+\log ^{+}\left|Z_{n}\right|\right)^{2} \geq n\right\}$ by $A_{n}$ and $\left\{\left(1+\log ^{+}\left|Z_{n}\right|\right) \leq\right.$ $\log n\}$ by $B_{n}$, respectively.

In terms of $A_{n}$ and $B_{n}$,

$$
P\left\{\frac{Z_{n}^{4}}{\left(1+\log ^{+}\left|Z_{n}\right|\right)^{2}} \geq n\right\}=P\left(A_{n} \cap B_{n}\right)+P\left(A_{n} \cap B_{n}^{C}\right) .
$$

So, $\sum_{n} P\left(A_{n}\right) \leq \sum_{n} P\left(A_{n} \cap B_{n}\right)+\sum_{n} P\left(B_{n}^{C}\right)$ and now consider

$$
\begin{aligned}
\sum_{n} P\left(B_{n}^{C}\right)= & \sum_{n} P\left(\log ^{+}\left|Z_{n}\right|>\log n-1\right) \\
= & \sum_{n} P\left(\log ^{+}\left|Z_{n}\right|>\log n-1,\left|Z_{n}\right| \leq 1\right) \\
& +\sum_{n} P\left(\log ^{+}\left|Z_{n}\right|>\log n-1,\left|Z_{n}\right|>1\right) \quad \text { for } n \geq 3 .
\end{aligned}
$$


The first event is an impossible event, whereas the second term on the right is

$$
\sum_{n} P\left(\log ^{+}\left|Z_{n}\right|>\log n-1,\left|Z_{n}\right|>1\right) \leq \sum_{n} P\left(\left|Z_{n}\right|>\alpha n\right)<\infty \quad \text { for some } \alpha>0
$$

if and only if $E\left|Z_{n}\right|<\infty$ which is true as a result of $E\left(Z^{2}\right)=d^{2}<\infty$. Hence, we require that $\sum_{n} P\left(A_{n} \cap B_{n}\right)<\infty$, which is satisfied if and only if $E\left[Z^{4} /\left(1+\log ^{+}|Z|\right)^{2}\right]<\infty$. Then, by Borel-Cantelli lemma, we have $P\left(A_{n}\right.$, i. o. $)=0$. This implies

$$
\frac{Z_{n}}{n^{1 / 4} \log ^{1 / 2} n} \longrightarrow 0 \quad \text { a.s. }
$$

This is the same as saying $Z_{n}=o\left(n^{1 / 4} \log ^{1 / 2} n\right)$ a.s. In particular, $Z_{n}=o(\sqrt{n})$ a.s. This means that for a sequence of i.i.d. random variables $\left\{Z_{i}\right\}_{i \geq 1}$ with Tauberian condition $Z_{n}=o\left(n^{1 / 4} \log ^{1 / 2} n\right)$ a.s., any two random-walk methods are equivalent. We could also show that under the same Tauberian condition, any random-walk method in (II) and any convolution summability method in (III) are equivalent. But for $Z_{n}=o(\sqrt{n})$, the result holds as $Z_{n}=o\left(n^{1 / 4} \log ^{1 / 2} n\right)$ implies $Z_{n}=o(\sqrt{n})$ and, the two methods are equivalent.

Suppose (I) holds, then (II) holds. Since $Z_{j}=o\left(j^{1 / 4} \log ^{1 / 2} j\right)$ a.s. for all $j \geq 1$ and (II) implies (III), we have that (I) implies (III) for a particular convolution summability method. (III) implies (II) will be shown similarly. The following two inequalities will assist in justifying these two assertions:

$$
\begin{aligned}
& P\left\{\limsup _{n \rightarrow \infty}\left|(4 \pi n)^{1 / 4}\left(d^{2} \log n\right)^{-1 / 2}\right| \sum_{j=0}^{\infty} R_{n, j} Z_{j}\left|-\frac{1}{\sigma^{1 / 2}}\right| \geq \epsilon\right\} \\
& \leq P\left\{\limsup _{n \rightarrow \infty}\left|(4 \pi n)^{1 / 4}\left(d^{2} \log n\right)^{-1 / 2}\right| \sum_{j=0}^{\infty} C_{n, j} Z_{j}\left|-\frac{1}{\sigma^{1 / 2}}\right| \geq \epsilon\right\} \\
& +P\left\{\limsup _{n \rightarrow \infty}\left|(4 \pi n)^{1 / 4}\left(d^{2} \log n\right)^{-1 / 2}\right|\right. \\
& \left.\quad \times\left|\sum_{j=0}^{\infty} R_{n, j} Z_{j}\right|-(4 \pi n)^{1 / 4}\left(d^{2} \log n\right)^{-1 / 2}\left|\sum_{j=0}^{\infty} C_{n, j} Z_{j}\right| \geq \epsilon\right\}, \\
& P\left\{\limsup _{n \rightarrow \infty}\left|(4 \pi n)^{1 / 4}\left(d^{2} \log n\right)^{-1 / 2}\right| \sum_{j=0}^{\infty} C_{n, j} Z_{j}\left|-\frac{1}{\sigma^{1 / 2}}\right| \geq \epsilon\right\} \\
& \leq P\left\{\limsup _{n \rightarrow \infty}\left|(4 \pi n)^{1 / 4}\left(d^{2} \log n\right)^{-1 / 2}\right| \sum_{j=0}^{\infty} R_{n, j} Z_{j}\left|-\frac{1}{\sigma^{1 / 2}}\right| \geq \epsilon\right\} \\
& \quad+P\left\{\limsup _{n \rightarrow \infty}\left|(4 \pi n)^{1 / 4}\left(d^{2} \log n\right)^{-1 / 2}\right|\right. \\
& \left.\quad \times\left|\sum_{j=0}^{\infty} C_{n, j} Z_{j}\right|-(4 \pi n)^{1 / 4}\left(d^{2} \log n\right)^{-1 / 2}\left|\sum_{j=0}^{\infty} R_{n, j} Z_{j}\right| \geq \epsilon\right\} .
\end{aligned}
$$


As we have seen before, for a sequence $\left\{Z_{i}\right\}_{i \geq 1}$ satisfying a Tauberian condition $Z_{j}=o(\sqrt{j})$ a.s. for all $j \geq 1$, any two random-walk methods are equivalent. Since $\sqrt{j} \geq$ $j^{1 / 4} \log ^{1 / 2} j$ for all $j \geq 2$, the equivalence of these two methods holds for a sequence of random variables with Tauberian condition $Z_{j}=o\left(j^{1 / 4} \log ^{1 / 2}\right)$ a.s. for all $j \geq 1$. And also for this Tauberian condition any random-walk method and any convolution method are equivalent. Hence the equivalence of (II) and (III) hold true not only for a particular method, but also for any other method under consideration.

Remark 5.2. In conclusion, all members of the convolution summability methods; Euler, Borel, Taylor, Meyer-König methods, and so forth obey the LSL.

\section{Acknowledgments}

This paper stems from the author's Ph.D. dissertation [11] supervised by Professor M. Kazim Khan at the Kent State University, Kent, Ohio. The author wishes to thank him and the Department of Mathematics and Computer Science of the Kent State University for making it possible to undertake a degree program in mathematics. Thanks are also due to two unknown referees for their valuable comments and suggestions, and for pointing out some writing errors on earlier versions of this manuscript. They were immensely helpful in improving this paper to a greater extent.

\section{References}

[1] A. Bikjalis and G. Jasjunas, Limit theorems in the metric of the spaces $L_{1}$ and $l_{1}$, Litovsk. Mat. Sb. 7 (1967), 195-218.

[2] N. H. Bingham, On Valiron and circle convergence, Math. Z. 186 (1984), no. 2, 273-286.

[3] - Moving averages, Almost Everywhere Convergence (Columbus, Ohio, 1988), Academic Press, Massachusetts, 1989, pp. 131-144.

[4] N. H. Bingham and M. Maejima, Summability methods and almost sure convergence, Z. Wahrsch. Verw. Gebiete 68 (1985), no. 3, 383-392.

[5] Y. S. Chow, Delayed sums and Borel summability of independent, identically distributed random variables, Bull. Inst. Math. Acad. Sinica 1 (1973), no. 2, 207-220.

[6] Y. S. Chow and T. L. Lai, Limiting behavior of weighted sums of independent random variables, Ann. Probability 1 (1973), 810-824.

[7] Y. S. Chow and H. Teicher, Probability Theory: Independence, Interchangeability, Martingales, 2nd ed., Springer Texts in Statistics, Springer-Verlag, New York, 1988.

[8] Y. Déniel and Y. Derriennic, Sur la convergence presque sure, au sens de Cesàro d'ordre $\alpha, 0<\alpha<$ 1 , de variables aléatoires indepéndantes et identiquement distribuées, Probab. Theory Related Fields 79 (1988), no. 4, 629-636.

[9] W. Feller, An Introduction to Probability Theory and Its Applications. Vol. II, 2nd ed., John Wiley \& Sons, New York, 1966.

[10] V. F. Gaposhkin, Summation of weakly dependent sequences by Cesàro methods, Anal. Math. 13 (1987), no. 4, 269-279.

[11] R. Goonatilake, On probabilistic aspects of summability theory, Ph.D. dissertation, Department of Mathematics and Computer Science, Kent State University, Ohio, 1997.

[12] A. Gut, Complete convergence and Cesàro summation for i.i.d. random variables, Probab. Theory Related Fields 97 (1993), no. 1-2, 169-178.

[13] G. H. Hardy, Divergent Series. Vol. 25, Clarendon Press, Oxford, 1949. 


\section{Tauberian theorems for summability transforms}

[14] B. Heinkel, An infinite-dimensional law of large numbers in Cesàro's sense, J. Theoret. Probab. 3 (1990), no. 4, 533-546.

[15] H. Kesten, Sums of independent random variables—without moment conditions, Ann. Math. Statist. 43 (1972), 701-732.

[16] M. K. Khan, Statistical methods in analysis I: some Tauberian theorems for absolute summability, Pak. J. Statist. 7 (1991), no. 1, 21-32.

[17] T. L. Lai, Summability methods for independent, identically distributed random variables, Proc. Amer. Math. Soc. 45 (1974), no. 2, 253-261.

[18] G. G. Lorentz, Borel and Banach properties of methods of summation, Duke Math. J. 22 (1955), $129-141$.

Rohitha Goonatilake: Department of Mathematical and Physical Sciences, Texas A\&M International University, Laredo, TX 78041-1900, USA

E-mail address: harag@tamiu.edu 


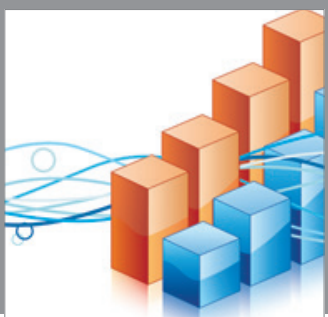

Advances in

Operations Research

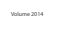

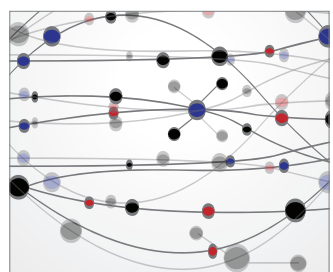

\section{The Scientific} World Journal
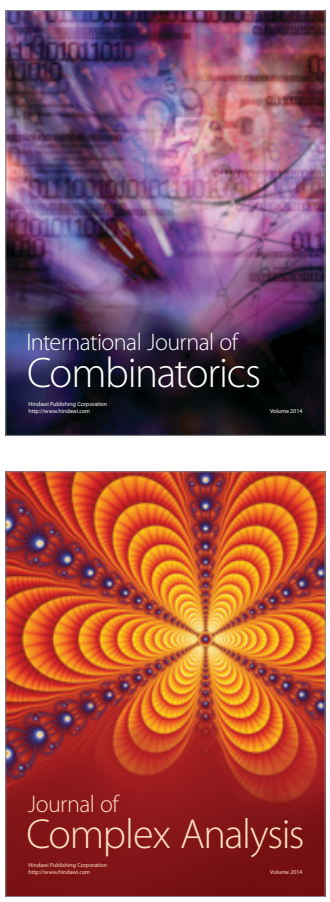

International Journal of

Mathematics and

Mathematical

Sciences
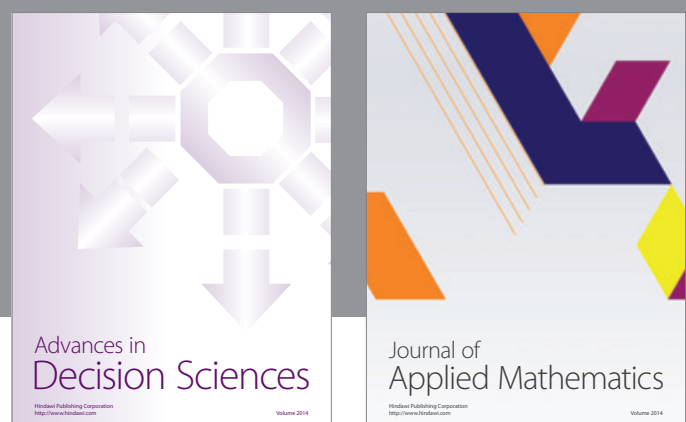

Journal of

Applied Mathematics
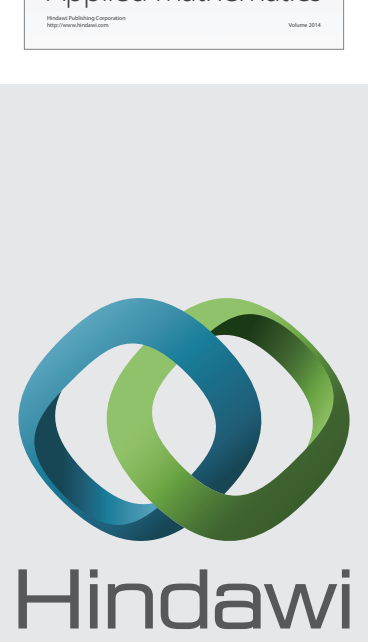

Submit your manuscripts at http://www.hindawi.com
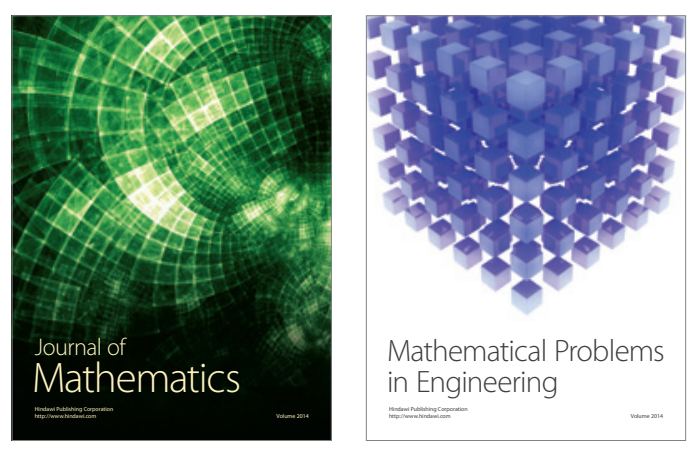

Mathematical Problems in Engineering
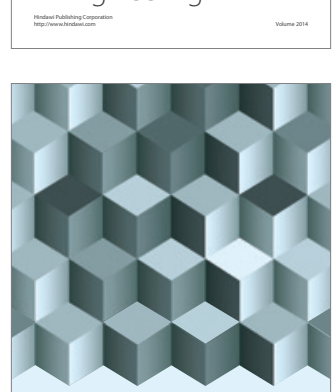

Journal of

Function Spaces
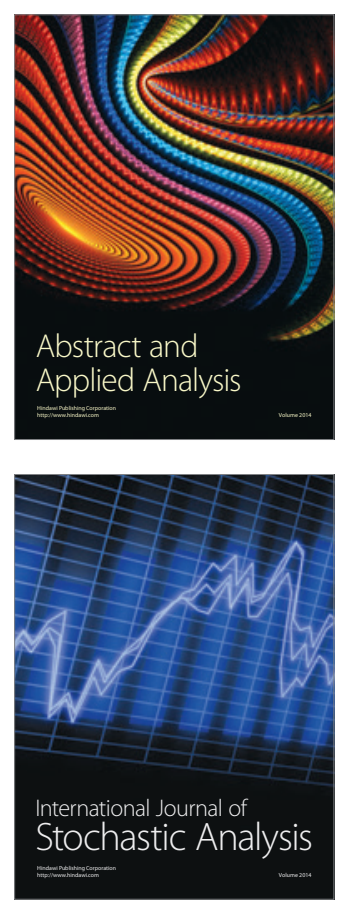

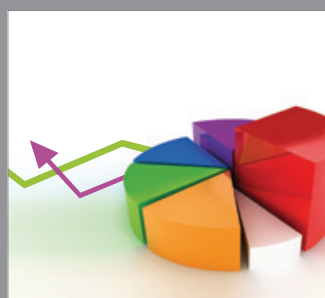

ournal of

Probability and Statistics

Promensencen
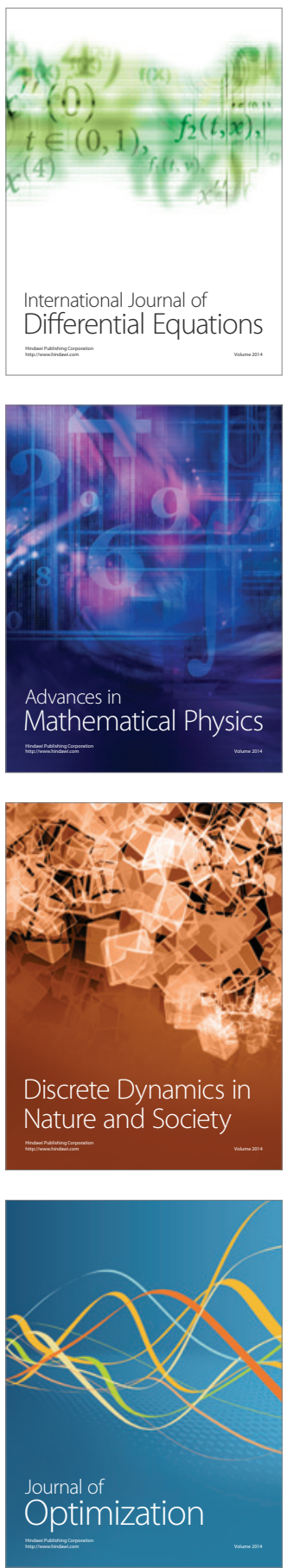